\title{
Prevalencia de síndrome metabólico en población laboral. El corazón de Asepeyo
}

\author{
J. GUTIÉRREZ GUISADO, J. J. LÓPEZ MANZANO ${ }^{1}$, J. RODRÍGUEZ CID¹, \\ C. GARCÉS SEGURA ${ }^{2}$, M. T. LLORENS RUFACH ${ }^{1}$ \\ Servicio Medicina Interna. Hospital Asepeyo. ${ }^{1}$ Servicio Prevención Propio Mutua \\ Asepeyo. ${ }^{2}$ Unidad de Lípidos Fundación Jiménez Díaz. Madrid
}

PREVALENCE OF METABOLIC SYNDROME (MS) IN LABORAL POPULATION. THE HEART OF ASEPEYO

\section{RESUMEN}

Introducción: El espectacular aumento que el síndrome metabólico (SM) está teniendo en las sociedades occidentales, conduce inevitablemente al incremento de la enfermedad cardiovascular. Por ello y puesto que su presencia indica la necesidad de aplicar medidas activas para reducir el riesgo cardiovascular, nos propusimos estudiar la prevalencia del síndrome metabólico y los factores de riesgo asociados a él, en nuestra población laboral.

Material y métodos: El estudio incluyó 345 trabajadores, 191 mujeres y 154 hombres, sometidos a un reconocimiento laboral durante el año 2006.

Resultados: El 12,5\% de la población presentó obesidad, siendo esta mayor en hombres $(16,9 \%)$ que en mujeres $(8,9 \%)$. La prevalencia de SM fue del 7,8\%, siendo mayor en hombres que en mujeres. El 57,7\% de los hombres y el 29,4\% de las mujeres con obesidad presentaban SM. Los trabajadores con más edad, tuvieron mayor prevalencia de SM. El criterio de SM más prevalente fue la hipertensión arterial. Todos los criterios de SM predominaron en varones a excepción del perímetro abdominal, que lo hizo en las mujeres.

Conclusiones:

1. Casi 8 de cada 100 trabajadores presentaron SM, siendo mayor la prevalencia en los varones. La obesidad aumentó de forma importante la prevalencia de SM. Los trabajadores con más edad, tuvieron mayor prevalencia de SM.

2. El perímetro abdominal se asoció significativamente con todos los factores de riesgo relacionados con el SM con excepción de la glucosa elevada en los hombres.

PALABRAS CLAVE: Perímetro abdominal. Prevalencia. Sindrome Metabólico. Población laboral.

\begin{abstract}
Background: The high increase of the metabolic syndrome (MS) on the occidental World, is increasing the cardiovascular disease. Since, as the presence of metabolic syndrome suggests the application of preventive measure neccesary, We studied, the prevalence of metabolic syndrome and the cardiovascular risk factors $(R F)$ in our laboral population.

Methods: The sample included 345 workers, 191 females and 154 males. All the people passed a laboral chec-up at the year 2006.

Results: Obesity was presented in $12.5 \%$ of the sample, $16.9 \%$ in males, $8.9 \%$ in females. The prevalence of MS was $7.8 \%$, being higher in males than in females. $57.7 \%$ of obeses males presented MS and $29.4 \%$ of obeses females presented MS. The older worker presented higher prevalence of MS. The high blood pressure was the factor more prevalent, in worker with MS. All the factors of MS were more pevalent in males, to exception of waist circumference.

Conclusions:

1. Almost $8 \%$ of workers presented MS, being higher the prevalanece in males. The obesity increased of important manner the MS. The older worker had higher prevalence of MS.

2. The waist circumference associated with all the FR related with MS.
\end{abstract}

KEY WORDS: Waist circumference. Prevalence. Metabolic Syndrome. Laboral population.

Gutiérrez Guisado J, López Manzano JJ, Rodríguez Cid J, Garcés Segura C, Llorens Rufach MT. Prevalencia de síndrome metabólico en población laboral. El corazón de Asepeyo. An Med Interna (Madrid) 2008; 25: 325-330.

\section{INTRODUCCIÓN}

La obesidad es una enfermedad que se caracteriza por el exceso de grasa corporal. En función de la grasa corporal, podríamos definir como sujetos obesos a aquellos que presentan porcentajes de grasa corporal por encima de los valores considerados normales, que son del 12 al $20 \%$ en varones y del 20 al $30 \%$ en mujeres adultas (1). El índice de masa corporal (IMC) es el índice recomendado por diversas sociedades médicas para valorar la adiposidad en la mayoría de la población. En España se siguen las recomendaciones de la Sociedad Española para el Estudio de la Obesidad (SEEDO), que se establecieron en el año 1996 (2), que introdujo algunas variaciones a la clasificación propuesta por la Organización Mundial de la Salud (OMS) (3). El peso normal, varía entre $18,5-24,9 \mathrm{~kg} / \mathrm{m}^{2}$, el sobrepeso comprende entre $25-29,9 \mathrm{~kg} / \mathrm{m}^{2}$ y la obesidad se define a partir de 30 
$\mathrm{kg} / \mathrm{m}^{2}$. Así la prevalencia de obesidad en la población adulta de 25 a 64 años según el estudio DORICA (4), se estima en un $15,5 \%$, y es más elevada en mujeres $(17,5 \%)$ que en varones $(13,2 \%)$. Y lo que es más importante está adquiriendo un comportamiento de epidemia, al ver incrementada su prevalencia en los últimos 14 años en un 34,5\% (5).

La relación de la obesidad, con la hipertensión ( HTA), la dislipemia y la diabetes mellitus, es decir, con los distintos factores de riesgo (FR) cardiovascular es bien conocida (6). Ya Kylin, médico sueco, describió a principios del siglo XX, la asociación entre HTA, hiperglucemia y gota. Posteriormente Marañón señaló de manera explícita a la HTA y a la obesidad como estados prediabéticos, y que debería exisitir una predisposición para la asociación entre la obesidad, la HTA, la diabetes e incluso la gota (7). Fue en 1947, cuando Vague destacó la asociación de la obesidad central (androide o masculina) con una serie de alteraciones metabólicas, que se encontraban en la diabetes y en la enfermedad cardiovascular (ECV) (8). Reaven (9) describió en 1988 la presencia de un conjunto de alteraciones metabólicas cuyo rasgo fisiopatológico central era la resistencia a la insulina. Denominó a este cuadro "síndrome X", que asociaba junto a ésta, intolerancia a la glucosa, hiperinsulinemia, aumento de triglicéridos en las lipoproteínas de muy baja densidad (VLDL), disminución del colesterol de las lipoproteínas de alta densidad (HDL), e HTA, pero, de manera sorprendente, no incluyó la obesidad en él; sin embargo, la obesidad se ha recogido en el concepto de Síndrome Metabólico (SM ) en todas las definiciones posteriores de la OMS (10), el grupo EGIR (European Group for the Study of Insulin Resistance) (11), el Programa Norteamericano para la Detección, Evaluación y Tratamiento de la Hipercolesterolemia en adultos (ATP III) (12), y la Federación Internacional de Diabetes (IDF) (7).

La definición de la OMS y del EGIR mantenían la resistencia frente a la insulina como un componente esencial, dado que se consideraba que dicha resistencia constituía el principal determinante etiológico del síndrome metabólico. La definición del EGIR también introdujo el perímetro de la cintura $(94 \mathrm{~cm}$ en los varones y $80 \mathrm{~cm}$ en las mujeres) como medida de la adiposidad.

Dos años después, el NCEP (National Cholesterol Education Program) introdujo la definición ATP-III. Propuesta para su aplicación en la práctica clínica, esta definición no incluía una cuantificación específica de la sensibilidad a la insulina y adoptó un abordaje menos "glucocéntrico", considerando por igual todos los componentes del síndrome metabólico. El parámetro de cuantificación de la obesidad seguía siendo el perímetro de la cintura, aunque con valores umbral superiores a los utilizados en la definición del EGIR (102 cm en los varones y $88 \mathrm{~cm}$ en las mujeres). La definición ATP-III alcanzó una gran popularidad debido a su sencillez. No obstante, a diferencia de lo que ocurría con la definición de la OMS, la definición ATP-III no incorporaba variables proinflamatorias ni protrombóticas como parte de una definición ampliada.

La IDF estableció una nueva definición, en la que la obesidad central representa un requisito necesario y en la que, por primera vez, se ofrecen valores umbral para definir la obesidad que son diferentes en los distintos grupos étnicos (7).

En la actualidad se denomina SM al conjunto de alteraciones metabólicas constituido por la obesidad de distribución central, la disminución de las concentraciones del colesterol unido a las lipoproteínas de alta densidad (cHDL), la elevación de las concentraciones de triglicéridos, el aumento de la tensión arterial (TA) y la hiperglucemia (7).
El SM se está convirtiendo en uno de los principales problemas de salud pública del siglo XXI, asociado a un incremento de 5 veces en la prevalencia de diabetes tipo 2 y de 2-3 veces en la de ECV (6) y se considera que el SM es un elemento importante en la epidemia actual de diabetes y de ECV, de manera que se ha convertido en un problema de salud pública importante en todo el mundo (6).

Nosotros en nuestro medio, que es una Unidad Básica de Salud en Madrid, del Servicio de Prevención Propio (SPP) de una Mutua de Accidentes Laborales y Enfermedades Profesionales de la Seguridad Social, hemos venido observando en los últimos años un aumento de la prevalencia del sobrepeso, de la hiperglucemia y de la HTA entre nuestros trabajadores, y por ello nos propusimos realizar un estudio epidemiológico de nuestra población y describir la prevalencia de SM según los criterios del ATP III (12).

\section{MATERIAL Y MÉTODOS}

\section{POBLACION DE ESTUDIO}

Se incluyeron en el estudio 345 personas, 191 mujeres y 154 hombres, a los que se les realizó el reconocimientos médico laboral durante el año 2006, por parte del SPP (Servicio Prevención Propio) de la Mutua que incluye a la población laboral de los centros asistenciales de la provincia de Madrid (Alcalá de Henares, Alcobendas, Arganda del Rey, Coslada, Fuenlabrada, Getafe, Las Rozas, Legazpi, Madrid Centro, Ciudad Lineal, Villaverde Alto, Mercamadrid, Móstoles, Pinto, Torrejón de Ardoz, Tres Cantos, Valdemoro), Avila, Segovia, Toledo, Guadalajara ,Valdepeñas y Puertollano.

\section{RECOGIDA DE INFORMACIÓN}

El reconocimiento constaba de una historia clínica, donde se recogió la edad, el sexo, si realizaban ejercicio físico (al menos $1 / 2$ hora al día 3 días a la semana), y si fumaban (cualquier número de cigarrillos). Se recogió información sobre las medidas antropométricas y la tensión arterial. La talla se midió hasta el último milímetro y el peso hasta el último 0,1 $\mathrm{kg}$, utilizando una balanza digital homologada que lleva incorporada un estadiómetro. Se pesó a los trabajadores sin zapatos y con ropa ligera. A partir de estas medidas se calculó el índice de masa corporal (IMC) (peso en $\mathrm{kg} / \mathrm{talla}^{2}$ en $\mathrm{m}^{2}$ ) y se determinó la prevalencia de sobrepeso y obesidad calculando el porcentaje de sujetos que superaban los puntos de corte de IMC que definen estos parámetros (sobrepeso para IMC a partir de $25 \mathrm{~kg} / \mathrm{m}^{2}$ y obesidad a partir de $30 \mathrm{~kg} / \mathrm{m}^{2}$ ). Asi mismo, se midió el perímetro abdominal hasta el último milímetro con una cinta métrica, tomando como referencia, la circunferencia medida desde la espina ilíaca y el margen costal inferior. La toma de la Tensión Arterial (TA), fue realizada siempre por el mismo enfermero del SPP, con un esfingomanómetro portátil homologado.

A los sujetos incluidos en el estudio se les realizó una extracción de sangre por venopunción después de 8 horas de ayuno. Se valoraron por métodos enzimáticos estandarizados colesterol y triglicéridos en un autoanalizador Hitachi. El colesterol unido a lipoproteínas de alta densidad (c-HDL) se determinó después de la precipitación de las lipoproteínas que 
contienen apo B con ácido fosfowolfrámico y $\mathrm{Mg}$. El colesterol unido a lipoproteínas de baja densidad (c-LDL) se calculó según la formula de Friedewald.

Para la definición de síndrome metabólico (SM) se utilizaron los criterios del ATP III (12). Se considera que existe SM cuando se cumplen 3 o más de los siguientes criterios:

-Obesidad abdominal $>102 \mathrm{~cm}$ en varones $\mathrm{y}>88 \mathrm{~cm}$ en mujeres.

- Hipertrigliceridemia $>$ o igual de $150 \mathrm{mg} / \mathrm{dl}$.

-Colesterol HDL $<40 \mathrm{mg} / \mathrm{dl}$ en varones o $<50 \mathrm{mg} / \mathrm{dl}$ en mujeres.

- Tensión arterial > o igual de 130/85 mmHg.

- Glucemia basal > o igual de $110 \mathrm{mg} / \mathrm{dl}$.

\section{MÉTODO ESTADÍSTICO}

La comparación de las frecuencias entre grupos se realizó mediante un test $\mathrm{Chi}^{2}$. El contraste estadístico de las diferencias se ha realizado mediante t-test en el caso de comparaciones entre dos grupos y mediante análisis ANOVA para la comparación entre sujetos normales, con sobrepeso y obesos. Para variables que no siguieron una distribución normal, los análisis estadísticos se llevaron a cabo después de su transformación logarítmica. Los análisis se realizaron con el programa SPSS versión 9.0.

\section{RESULTADOS}

Se analiza una población total de 345 trabajadores, 154 hombres y 191 mujeres.

En el análisis descriptivo de las variables estudiadas en hombres y mujeres, se encontró unas cifras más elevadas de todas las variables en hombres con respecto a las mujeres, a excepción del c-HDL que estaba más bajo en los hombres.

En la tabla I se describen las prevalencias de los criterios que definen el SM según los criterios del ATP III, junto con la realización de ejercicio y el hecho de fumar. Se encuentra que el parámetro más alterado es la TA, mostrando la elevación de la TA diastólica una prevalencia del $20,3 \%$, y siendo la de TA sistólica del $22 \%$. El c-HDL y el perímetro abdominal son los siguientes criterios más frecuentemente alterados, alcanzando el 20,8 y el 18,6\% respectivamente. El menos prevalente sería la glucemia en ayunas con un 5,2\%. En los hombres es la TA sistólica el criterio más alterado con el $37,6 \%$ y en las mujeres el perímetro abdominal, con el $25,1 \%$.

Fuman el 18,3\% con predominio de las mujeres y realizan ejercicio el $42,9 \%$ con predominio franco de los varones.

Nuestro estudio presenta un porcentaje de sobrepeso en hombres y mujeres del 46,8 y del $23,6 \%$, respectivamente. El porcentaje de obesidad es del $16,9 \%$ en hombres y $8,9 \%$ en mujeres. El porcentaje total de sobrepeso es $33,9 \%$, y el de obesidad $12,5 \%$.

En las tablas II y III se analizan las distintas variables en hombres y mujeres, normales, con sobrepeso (IMC > 25) y obesidad (IMC > 30). Tanto en hombres y mujeres, encontramos en todas las variables estudiadas una diferencia significativa entre el grupo de personas normales y las obesas, a excepción de la glucosa, en donde no hay diferencia significativa ni en hombres ni mujeres, si bien, en mujeres existe una diferencia significativa entre las normales y las que tienen sobrepeso.

\section{TABLA I}

PREVALENCIA DE LOS FR QUE DEFINEN EL SM EN POBLACIÓN TOTAL, HOMBRES Y MUJERES

\begin{tabular}{lccc}
\hline & Total (\%) & Hombres (\%) & Mujeres (\%) \\
\hline TAS & 22 & 37,6 & 9,4 \\
TAD & 20.3 & 26,6 & 15,18 \\
Perímetro Abdominal & 18,6 & 10,4 & 25,1 \\
Clucemia & 5,2 & 10,4 & 1 \\
c-HDL & 20,08 & 22,9 & 19,9 \\
TC & 11,9 & 18,2 & 6,8 \\
Fumar & 18,3 & 16,9 & 19,4 \\
Ejercicio & 42,3 & 55,2 & 31,9 \\
\hline
\end{tabular}

TG: triglicéridos; TAS: tensión arterial sistólica; TAD: tensión arterial diastólica.

\section{TABLA II}

ANÁLISIS COMPARATIVO DE LAS DISTINTAS VARIABLES EN HOMBRES NORMALES, CON SOBREPESO Y OBESOS

\begin{tabular}{lcccc}
\hline & Normales & Sobrepeso & Obesos & $P$ \\
\hline Edad (años) & $39,4(10,3)$ & $40(9,9)$ & $46,6(10)$ & $1-3^{*}$ \\
& & & & $2-3^{*}$ \\
TAS (mmHg) & $12,3(1,1)$ & $12,3(0,9)$ & $13,5(1,5)$ & $1-3^{*}$ \\
& & & & $2-3^{*}$ \\
TAD (mmHg) & $7,7(0,7)$ & $7,8(0,7)$ & $8,6(0,9)$ & $1-3^{* *}$ \\
& & & & $2-3^{* *}$ \\
IMC (kg/m²) & $23,1(1,5)$ & $26,8(1,3)$ & $33,3(2,5)$ & $1-3^{* *}$ \\
Perímetro cintura (cm) & $86,1(7,9)$ & $93,6(8)$ & $109,4(10,5)$ & $1-3^{* *}$ \\
Clucosa (mg/dl) & $93,5(36)$ & $92,7(14,6)$ & $99,1(24,9)$ & $\mathrm{NS}$ \\
CT (mg/dl) & $183,5(34,8)$ & $194,4(34,2)$ & $206,6(37,3)$ & $1-3^{*}$ \\
c-HDL (mg/dl) & $52,4(10,7)$ & $47,2(10,3)$ & $42(8,1)$ & $1-2^{*}$ \\
& & & & $1-3^{* *}$ \\
c-LDL (mg/dl) & $106,7(29,7)$ & $119,3(28,4)$ & $133,6(30,3)$ & $1-2^{*}$ \\
& & & & $1-3^{* *}$ \\
TC (mg/dl) & $91,5(53,5)$ & $110,1(58,9)$ & $155,1(78,1)$ & $1-3^{*}$ \\
& & & & $2-3^{*}$ \\
\hline
\end{tabular}

Los valores se expresan como media (desviación estándar); P: comparación estadística por el test de ANOVA; $p<0,05 ;{ }^{*} p<0,001 ; C T$ : colesterol total; TG: triglicéridos; TAS: tensión arterial sistólica; TAD: tensión arterial diastólica.

En los hombres, existen diferencias significativas en el grupo de personas con sobrepeso en relación a las obesas, con las variables de TA tanto sistólica como diastólica, y los TG. En las mujeres, existe esta diferencia significativa en el perímetro abdominal y los TG. Es decir conforme se aumenta de peso, se incrementa los valores de TA, perímetro abdominal, y los de las variables bioquímicas, excepto la glucosa en hombres.

El SM en personas obesas se sitúa en el 46,5\%, alcanzando en los hombres el $57,7 \%$ y en las mujeres el 29,4\%. En personas normales la media de SM es del 1,6\%. En población total, la prevalencia de SM es del 7,8\%, siendo en los hombres del $11,7 \%$ y en las mujeres del $5,7 \%$.

En la tabla IV se describen los porcentajes de los factores de riesgo del SM, junto con los del ejercicio y el hecho de fumar en población con y sin SM. En trabajadores con SM, la mayor prevalencia la comparten, el perímetro abdominal, la TA sistólica y el c-HDL alterados, con el $72,4 \%$, siendo la glucosa el criterio menos alterado, con el 34,5\%. En los hom- 
TABLA III

ANÁLISIS COMPARATIVO DE LAS DISTINTAS VARIABLES EN MUJERES NORMALES, CON SOBREPESO Y OBESAS

\begin{tabular}{lcccc}
\hline & Normales & Sobrepeso & Obesas & $P$ \\
\hline Edad (años) & $37(9,2)$ & $41,6(9,8)$ & $45,8(9,8)$ & $1-2^{*}$ \\
& & & & $1-3^{* *}$ \\
TAS (mmHg) & $10,9(1)$ & $11,6(1,3)$ & $12(0,9)$ & $1-2^{* *}$ \\
& & & & $1-3^{*}$ \\
TAD (mmHg) & $7(0,89)$ & $7,4(0,9)$ & $8,1(0,8)$ & $1-3^{* *}$ \\
IMC (kg/m²) & $21,7(1,9)$ & $26,6(1,3)$ & $33,1(2,4)$ & $1-2^{* *}$ \\
& & & & $1-3^{* *}$ \\
& & & & $2-3^{* *}$ \\
Perímetro cintura (cm) & $76,4(7,7)$ & $86,4(9,1)$ & $98,5(7,7)$ & $1-2^{* *}$ \\
& & & & $1-3^{* *}$ \\
& & & & $2-3^{* *}$ \\
Clucosa (mg/dl) & $83,6(8,4)$ & $90,6(11,9)$ & $87,2(9,6)$ & $1-2^{* *}$ \\
CT (mg/dl) & $180,5(32,6)$ & $190,6(34,3)$ & $206(51,1)$ & $1-3^{*}$ \\
C-HDL (mg/dl) & $64,6(13,4)$ & $55,1(11,7)$ & $52,4(9)$ & $1-2^{*}$ \\
& & & & $1-3^{* *}$ \\
c-LDL (mg/dl) & $97,9(26)$ & $110,7(28,9)$ & $121,7(40,5)$ & $1-2^{*}$ \\
& & & & $1-3^{* *}$ \\
TC (mg/dl) & $68,9(35,3)$ & $86,8(48,5)$ & $124,4(84,5)$ & $1-3^{*}$ \\
& & & & $2-3^{*}$ \\
\hline
\end{tabular}

Los valores se expresan como media (desviación estándar); P: comparación estadística por el test de ANOVA; $p<0,05 ;{ }^{*} p<0,001 ; C T$ : colesterol total; TG: triglicéridos; TAS: tensión arterial sistólica; TAD: tensión arterial diastólica.

bres es la TA sistólica el FR con mayor prevalencia, 72,2\%, y en mujeres el c-HDL y el perímetro abdominal, con el $81,8 \%$. En población sin SM son la TA junto con el c-HDL los FR con mayor prevalencia en torno al $17 \%$. Hay que tener en cuenta que los pacientes que presentan SM tienen mayor edad que los que no lo presentan.

Por otro lado, fuman más y hacen menos ejercicio las personas con SM, en comparación con las que no lo tienen. En población sin SM fuman más las mujeres.

En la tabla V, se muestra la relación del perímetro abdominal en hombres y mujeres con la TA, IMC, edad, y los parámetros bioquímicos estudiados y se aprecia que los que tienen

\section{TABLA IV}

PREVALENCIA DE LOS FR QUE DEFINEN EL SM. PORCENTAJE DE FUMADORES Y DE PERSONAS QUE REALIZAN EJERCICIO EN POBLACIÓN TOTAL Y EN HOMBRES Y EN MUJERES CON Y SIN SM

\begin{tabular}{|c|c|c|c|c|c|c|}
\hline & \multicolumn{3}{|c|}{ Si (\%) } & \multicolumn{3}{|c|}{ No (\%) } \\
\hline & Total & Hombres & Mujeres & Total & Hombres & Mujeres \\
\hline Clucosa & 34,5 & 44,4 & 18,2 & 1,6 & 3,7 & 0 \\
\hline$c-H D L$ & 72,4 & 66,7 & 81,8 & 16,52 & 19,1 & 17,2 \\
\hline $\mathrm{TC}$ & 69 & 66,7 & 72,7 & 6,6 & 11,8 & 2,8 \\
\hline PA & 72,4 & 66,7 & 81,8 & 13,6 & 2,9 & 21,7 \\
\hline TAS & 72,4 & 72,2 & 72,7 & 17,4 & 33 & 5,6 \\
\hline$T A D$ & 58,6 & 50 & 72,7 & 16,8 & 23,5 & 11,7 \\
\hline Fumar & 24,1 & 27,8 & 18,2 & 17,7 & 15,4 & 19,4 \\
\hline Ejercicio & 20,7 & 22,2 & 18,2 & 44,3 & 59,6 & 32,8 \\
\hline
\end{tabular}

PA: perímetro abdominal. TG: triglicéridos. TAS: tensión arterial sistólica; TAD: tensión arterial diastólica.
TABLA V

\section{RELACIÓN DEL PERÍMETRO ABDOMINAL ANORMAL, CON LAS DISTINTAS VARIABLES: IMC, TA, EDAD Y VARIABLES BIOQUÍMICAS EN MUJERES Y EN HOMBRES}

\begin{tabular}{lcccc}
\hline & Mujeres (1) & Hombres (2) & $P(1)$ & $P(2)$ \\
\hline EDAD (años) & $37,1(9,3)$ & $47,5(9,3)$ & $* *$ & $*$ \\
TAS (mmHg) & $10,9(1)$ & $13,8(1,7)$ & $* *$ & $*$ \\
TAD (mmHg) & $7(0,8)$ & $8,7(0,9)$ & $* *$ & $* *$ \\
IMC (kg/m²) & $22,4(2,4)$ & $33,1(3,9)$ & $* *$ & $* *$ \\
CLUCOSA (mg/dl) & $83,8(8,3)$ & $101,3(29,4)$ & $* *$ & NS \\
CT (mg/dl) & $181,2(33,5)$ & $216,4(43,7)$ & $*$ & $*$ \\
C-HDL (mg/dl) & $63,6(13,3)$ & $40,5(7,5)$ & $* *$ & $*$ \\
c-LDL (mg/dl) & $98(26,3)$ & $139,8(33,5)$ & $* *$ & $*$ \\
TC (mg/dl) & $70,4(36,6)$ & $159,1(74,5)$ & $*$ & $* *$
\end{tabular}

Los valores se expresan como media (desviación estándar); CT: colesterol total; TG: triglicéridos; TAS: tensión arterial sistólica; TAD: tensión arterial diastólica; p: comparación estadística entre 2 grupos, mediante ttest; ${ }^{*} p<0,05 ;{ }^{* *} p<0,001 ;(1)$ : Mujeres; (2): Hombres.

mayor perímetro abdominal, tienen los parámetros bioquímicos y antropométricos más alterados que los que no lo tienen y además las diferencias son significativas, a excepción de la glucosa en hombres.

\section{DISCUSIÓN}

Hipócrates describió: "Corpulence is not only a disease itself, but the harbinger of others", es decir, ya reconocía, que la obesidad es un desorden médico, que se asocia a muchas comorbilidades (13). Es bien conocido por la literatura internacional, que el riesgo de diabetes, HTA y dislipemia, se incrementa con la obesidad, y lo hace a partir de un IMC de $21 \mathrm{~kg} / \mathrm{m}^{2}$, reduciendo la expectativa de vida, junto con un incremento muy importante en los gastos de salud. En la actualidad, en el mundo, la obesidad es la $6^{\mathrm{a}}$ causa de carga económica debida a enfermedades (14).

En nuestra serie hemos encontrado una prevalencia de sobrepeso del $33.9 \%$ y de obesidad del $12,5 \%$. En mujeres la prevalencia de obesidad es del 8,9\% y en hombres del 16,9\%. Nuestros datos, concuerdan con la Encuesta Nacional de Salud (2001) (15), donde se refiere un incremento absoluto en la tasa de obesidad del 6\%, en los últimos 14 años, situándose la prevalencia en el $13,6 \%$, pero, son sensiblemente inferiores a los mostrados en el estudio DORICA (4), para población de 25 a 64 años, que es nuestro caso (con una media de edad de 40 años), con una prevalencia de obesidad del 15,5\%, si bien, la prevalencia es más elevada en mujeres que en hombres , 17,5 y $13,2 \%$ respectivamente, al contrario de lo que ocurre en nuestra serie, donde la prevalencia en los hombres es casi el doble, que en las mujeres. En los EE. UU., en población adulta en el 2005, se encontró una tasa de obesidad del $23,9 \%$, siendo la prevalencia en mujeres y hombres similar, 23,5\% (16). En Europa occidental la tasa de obesidad se encuentra entre $9,9 \%$ de países como Francia e Italia y el $24 \%$ de Finlandia (17).

Si comparamos con otros estudios en población laboral española, nuestra tasa de obesidad del $12,5 \%$, es claramente inferior, pues, varían entre el 15,5 y el $28 \%$, con un claro predominio de los varones en el estudio Mesyas (30 y $7 \%$ respectivamente) (18-20). 
El SPP de la Mutua asumiendo un aumento de la prevalencia del sobrepeso de nuestra población, puso en marcha en el 2006, una campaña para intentar disminuir el sobrepeso y sus resultados los estamos analizando en la actualidad.

En nuestro estudio, tanto en hombres como en mujeres la obesidad supone un aumento de TA, tanto sistólica como diastólica, de perímetro abdominal, y de los parámetros bioquímicos estudiados, cuya diferencia es estadísticamente significativa, salvo en los hombres en relación con la glucosa en ayunas. Hay que comentar, que en los hombres hay un caso de peso normal con una hiperglucemia franca (superior a 300), que es un diabético tipo I, con mal control, y que puede alterar algunos resultados. Si eliminamos este caso, la diferencia, casi, llegaría a ser significativa también en los hombres $(\mathrm{p}<0,09)$.

Como ya hemos comentado, estos hallazgos, se encuentran bien refrendados en la literatura internacional (14).

La prevalencia de los criterios diagnósticos de SM, en nuestra población es la siguiente:

- HTA 42,3\%.

-Perímetro abdominal 18,6\%.

- Glucemia 5,2\%.

-c-HDL 20,8\%.

- Triglicéridos $9 \%$.

Si comparamos nuestros resultados con los de un estudio reciente en poblacion laboral, que se realizó para determinar la prevalencia de SM, que es el estudio Mesyas (19) encontramos que las prevalencias en este último, de perímetro abdominal (estimado por IMC > 28,5), glucemia y TG, son claramente superiores $(28,5,7,5,18,3 \%)$, mientras que nuestra prevalencia de c-HDL es francamente superior $(20,8$ vs. 12,6\%). La prevalencia de HTA es similar en ambos estudios (42,3 vs. 40\%). Sin embargo comparando con otro estudio también reciente en población laboral (20), nuestras prevalencias son superiores, sobre todo a nivel de HTA , que doblamos su prevalencia; sin embargo el c-HDL es inferior (20,8 frente a $25 \%$ ).

En cuanto al sexo, en nuestro estudio la prevalencia de los FR para SM es mayor en los varones, al igual que en los estudios previos.

Comparando la prevalencia de los FR de nuestro estudio con población general en España (21) (estudio PANES) nosotros tenemos una prevalencia superior de HTA, y dislipemia e inferior de hiperglucemia.

Por tanto estamos en una situación intermedia en lo que se refiere a población laboral e incluso nos podemos equiparar al riesgo cardiovascular en población general, lo que es preocupante porque el rango de edad en población general es superior (45 a 74 años), y nos debe poner alertas, y comenzar con políticas de prevención cardiovascular de una forma activa.

La prevalencia de SM en nuestra población es de 7,8\%, siendo mayor en hombres que en mujeres 11,7 y $5,7 \%$ respectivamente. Cuando lo relacionamos con la obesidad, la prevalencia de SM se incrementa considerablemente, y así tenemos una prevalencia del $46,5 \%$, siendo mayor en hombres que en mujeres , 57,7\% y $29,4 \%$ respectivamente. A su vez, en nuestro estudio se aprecia que a mayor edad, mayor obesidad, y por tanto mayor prevalencia de SM, asociándose éste de forma significativa con la edad. En otros estudios en población laboral, encontramos unos resultados similares, como en el estudio de Sánchez Chaparro (20), donde también se situó la prevalencia en el 10\%. En el estudio Mesyas (19), la prevalencia fue del $10 \%$, si bien, una vez se ajustó por edad y sexo se situó en el 5,8\%, siendo más alta en hombres que en mujeres,
8,7 y $3 \%$ respectivamente. Además la prevalencia del SM aumentó de forma paralela con la edad y la obesidad. En otros estudios en población general en España, la prevalencia de SM según criterios ATP III es superior, variando del $14 \%$ al $24 \%$, en Segovia y Canarias respectivamente $(22,23)$, lo que es lógico, pues, se incluye a una población con mayor edad, y por tanto mayor prevalencia de SM.

En Europa,en otros estudios en población general, según el estudio DECODE, la prevalencia es del 15\% (criterios OMS) (24). En EE. UU., el estudio NHANES III (25), encontró una prevalencia con criterios ATP III, del 20\% al 25\%, siendo la prevalencia igual en hombres y mujeres, lo que es lógico pues con la menopausia de las mujeres, la prevalencia del SM experimenta un incremento mayor y gradual, hasta ser comparable a la de los varones alrededor de los 70 años.

En cuanto a la prevalencia de los FR en personas con SM encontradas en nuestro estudio, son la alteración del perímetro abdominal, el c-HDL y la HTA los más prevalentes, con 72, 72 y $65 \%$ respectivamente. En el estudio Mesyas (19) fue la HTA el FR más prevalente, con el $94.5 \%$.

En nuestro estudio fuma el $18 \%$ de la población, (algo más las mujeres que los hombres, $19,3 \%$ y $16,3 \%$, respectivamente) y realizan ejercicio el $42,3 \%$ de la población, siendo los hombres los que más ejercicio realizan. En los estudios en población laboral española (18-20), los fumadores, son claramente superiores, con una variación del 44 al 51\%, con predominio en los varones. En el estudio Mesyas (19), más del 50\% de la muestra son fumadores activos, y hombres y mujeres fuman en la misma proporción. Los fumadores se presentan en la misma proporción entre la población con o sin SM. El SM se asocia más con el hecho de ser exfumador, condición que también se aprecia en el estudio Whitehall (26). En población general los fumadores se sitúan en el 34\% con predominio de los varones (21). Desde luego la campaña llevada a cabo en el 2005 por el SPP en prevención del hábito tabáquico, tiene su influencia en estos hallazgos tan positivos, pues, de las personas fumadoras que se acogieron al programa, se ha reducido el hábito tábaquico en un $26 \%$ al cabo de los 2 años de seguimiento.

El $42 \%$ de la población estudiada, realiza ejercicio, al menos 30 minutos diarios 3 veces a la semana. En los estudios en población laboral, no hemos encontrado referencias, en cuanto a la realización de ejercicio, salvo en el estudio de Martínez González (18), que refiere una tasa de ejercicio (definido por realizar trabajos de esfuerzo o realizar algún deporte) del $82 \%$, siendo los varones los que más ejercicio realizan. En este estudio, la prevalencia de ejercicio es mayor que en el nuestro, porque los parámetros que lo definen son distintos, y no es del todo comparable. En un estudio sobre sedentarismo realizado en las Islas Canarias, se estima que el 40-55\% de los hombres y el 30\% de las mujeres, realizan ejercicio físico al menos 25 minutos diarios, relacionando el sedentarismo con el SM y los distintos FR cardiovasculares (27). Viendo estos resultados, uno de los próximos objetivos, que podríamos plantearnos sería una promoción del deporte y del ejercicio físico, para mejorarlos.

El aumento del sobrepeso y la obesidad en los países occidentales y su relación con las enfermedades cardiovasculares, ha hecho que adquiera el rango de principal problema sanitario de carácter epidémico (28). En nuestro país, la situación es similar, pues, si bien tenemos una prevalencia de obesidad intermedia dentro de los países occidentales, esta, ha aumentado en un $34.5 \%$ en los últimos 14 años (5). 
Los resultados de Ascaso et al (29) muestran el importante valor predictivo de la obesidad abdominal en la aparición de la resistencia insulínica y SM. En el estudio Mesyas (19), se comprobó que la prevalencia de obesidad aumentaba de forma importante la prevalencia de SM. En nuestra serie, analizamos el aumento del perímetro abdominal en relación con las distintas variables antropométricas y bioquímicas, TA y edad y tanto en hombres como en mujeres, hay un aumento en todas las variables, que es estadísticamente significativo, a excepción de la glucosa en los hombres. Cuando se elimina el caso de hiperglucemia con peso normal, casi la glucosa alcanza también la significación estadística. Es decir el aumento del perímetro abdominal se asocia con los distintos FR que definen el SM, IMC, y la edad.

Por tanto proponemos que la medida del perímetro abdominal, se incorpore en los reconocimientos médicos de los
Servicios de Prevención, porque nos puede avisar del riesgo cardiovascular del paciente de una forma muy sencilla, y comenzar a aplicar las medidas de prevención cardiovascular, lo más rápidamente posible: actividad física, dieta equilibrada y pérdida de peso, que son las medidas básicas para el manejo de los distintos FR cardiovasculares, y por tanto del SM sin implicar un coste económico elevado (30).

\section{AGRADECIMIENTOS}

El artículo va dedicado In Memoriam al Profesor Manuel de Oya Otero, que dedicó todas su vida al estudio de los lípidos y de los factores de riesgo cardiovascular y que fue maestro de los que firmamos el trabajo.

\section{Bibliografía}

1. Salas-Salvadó J, Rubio MA, Barbany M, Moreno B y Grupo Colaborativo de la SEEDO. Consenso SEEDO 2007 para la evaluación del sobrepeso y la obesidad y el establecimiento de criterios de intervención terapéutica. Med Clin (Barc) 2007; 128: 184-96.

2. Sociedad Española para el Estudio de la Obesidad (SEEDO). Consenso español 1995 para la evaluación de la obesidad y para la realización de estudios epidemiológicos. Med Clin (Barc) 1996; 107: 782-7.

3. WHO. Programme of Nutrition, Family and Reproductive Health. Obesity. Preventing and managing the global epidemic. Report of a WHO consultation on obesity. Geneva, 3-5 June, 1997. Geneva: WHO; 1998.

4. Aranceta Bartrina J, Serra Majem L, Pérez Rodrigo C, Foz Sala M, Moreno Esteban B y Grupo Colaborativo SEEDO. Prevalencia de obesidad en España. Med Clin (Barc) 2005; 125: 460-6.

5. Rubio MA, Gómez de la Cámara A, Del Campo J, Jurado C, García JD, Gómez-Gerique JA, et al. Prevalencia de obesidad en España tras 14 años de seguimiento de la cohorte DRECE. Endocr Nutr 2006; 53 (Supl. 1): 86 .

6. Eckel RH, Grundy SM, Zimmet PZ. The metabolic syndrome. Lancet. 2005; 365: 1415-28.

7. Zimmet P, K. Alberti G y Serrano Ríos M. Una nueva definición mundial del síndrome metabólico propuesta por la Federación Internacional de Diabetes: fundamento y resultados. Rev Esp Cardiol 2005; 58: 13716.

8. Vague J. Sexual differentiation, a factor affecting the forms of obesity. Pres Med 1947; 30: 339-40.

9. Reaven G. Role of insulin resistance in human disease. Diabetes. 1988; 37: $1595-607$.

10. World Health Organization. Definition, diagnosis and classification of diabetes mellitus and its complications. Report of a WHO consultation. Geneve: WHO; 1999.

11. Balkau B, Charles MA. Comment on the provisional report from the WHO consultation. European Group for the Study of Insulin Resistance (EGIR). Diabet Med. 1999; 16: 442-3.

12. Executive Summary of The Third Report of The National Cholesterol Education Program (NCEP) Expert Panel on Detection, Evaluation, And Treatment of High Blood Cholesterol In Adults (Adult Treatment Panel III). JAMA. 2001; 285: 2486-2.

13. Haslam DW, James PW. Obesity. Lancet 2005; 366: 1197-209.

14. Ezzati M, Lopez AD, Rodgers A, Vander Hoorn S, Murray CJ. Comparative Risk Assessment Collaborating Group. Selected major risk factors and global and regional burden of disease. Lancet 2002; 360: 134760.

15. Gutiérrez Fisac JL, Regidor E, Banegas JR, Rodríguez Artalejo F. Prevalencia de obesidad en población adulta española: 14 años de crecimiento continuado. Med Clin (Barc) 2005; 124: 196-7

16. State-Specific prevalence of obesity Among Adults-United Status. 2005. MMWR. 2006; 55: 985-8.

17. IOTF (Internacional Obesity Task Force). Position paper september
2003. Obesity in Europe-2: waiting for a green light for health? Europe at the crossroads for diet and disease.

18. Martínez González MA, Bueno Cavanillas A, Fernández García MA, García Martín M, Delgado Rodríguez M, Gálvez Vargas R. Prevalencia de factores de riesgo cardiovascular en población laboral. Med Clin (Barc) 1995; 10: 321-6.

19. Alegría E, Cordero A, Laclaustra M, Grima A, León M, Casasnovas JA, et al. Prevalencia del síndrome metabólico en población laboral española: registro Mesyas. Rev Esp Cardiol 2005: 58: 797-806.

20. Sánchez-Chaparro MA, Román-García J, Calvo-Bonacho E, GómezLarios T, Fernández-Meseguer A, Sáinz-Gutiérrez J, et al. Prevalencia de factores de riesgo vascular en la población laboral española. Rev Esp Cardiol 2006; 59: 421-30

21. López-Bescós L, Cosín J, Elosua R, Cabadés A, Reyes M, Arós F, Diago JL, Asín E, Castro-Beiras A y Marrugat J. Prevalencia de angina y factores de riesgo cardiovascular en las diferentes comunidades autónomas de España: estudio PANES. Rev Esp Cardiol 1999; 52: 1045-56.

22. Martínez Larrad MT, González Sánchez JL, López A, Fernández Pérez C, Serrano Ríos M. Segovia Insulin Resistance Study Group. The metabolic syndrome in Spain, report of the Segovia Insulin Resistance Study Group. Diab Metab 2003; 29: 45.

23. Alvarez León EE, Ribas Barba L, Serra Majem L. Prevalencia del síndrome metabólico en la población de la comunidad Canaria. Med Clin (Barc) 2003; 120: 172-4.

24. Hu G, Oiao Q, Tuonmilehto J, Balkau B, Borch-Johnsen K, PyoralaK; DECODE Study Group. Prevalence of the metabolic syndrome and its relation to all cause and cardiovascular mortality in non diabetic European men and women. Arch Intern Med 2004; 164: 1066-76.

25. Park YW, Zhu S, Palaniappan L, Heshka S, Carnethon MR, Heymsfield SB. The metabolic syndrome: prevalence and associated risk factor findings in the US population from the Third National Health and Nutrition Examination Survey, 1988-1994. Arch Intern Med 2003; 163: 427 36.

26. Brunner EJ, Marmot MG, Nanchahal K, Stanfeld SA, Juneja M, Alberti $\mathrm{KG}$. Social inequality in coronary risk: central obesity and the metabolic syndrome. Evidence from the Whitehall II Study. Diabetologia 1997; 40: 1341-9.

27. Cabrera de León A, Rodríguez-Pérez MC, Rodríguez-Benjumeda LM, Anía-Lafuente B, Brito-Díaz B, Muros de Fuentes M, et al. Sedentarismo: tiempo de ocio frente a porcentaje de gasto energético. Rev Esp Cardiol. 2007; 60: 244-50.

28. Haffner S, Taegtmeyer H. Epidemic obesity and the metabolic syndrome. Circulation 2003; 108: 1541-5.

29. Ascaso JF, Romero P, Real JT, Lorente RI, Martínez-Valls J, Carmena R. Abdominal obesity, insulin resistance, and metabolic syndrome in a southern European population. Eur J Intern Med 2003; 14: 101-6.

30. American Diabetes Association. Standards of medical care in diabetes. Diabetes Care 2004; 27 (Supl. 1): 21-3. 